\title{
ASSENTAMENTOS DE REFORMA AGRÁRIA E A VIGILÂNCIA EM SAÚDE PARA LEISHMANIOSE VISCERAL NA REGIÃO DE PRESIDENTE PRUDENTE-SP, BRASIL
}

\author{
Patrícia Ferreira da Silva ${ }^{1}$, Chelsea Pereira de Souza ${ }^{1}$, Matheus de Carvalho Moreno ${ }^{2}$, Lourdes Aparecida \\ Zampieri D' Andrea ${ }^{1,2}$ \\ ${ }^{1}$ Instituto Adolfo Lutz, Centro Laboratório Regional, Presidente Prudente, SP. ${ }^{2}$ Universidade Estadual Paulista - UNESP, \\ Faculdade de Ciências e Tecnologia, Presidente Prudente, SP. email: patriciaferreirabiomedica@gmail.com
}

\section{RESUMO}

A Leishmaniose Visceral (LV) possui interdependência com regiões de florestas remanescentes modificadas, e os espaços rurais tendem a armazenar vetores e reservatórios. Esse estudo objetivou dimensionar diferentes situações de risco epidemiológico conforme a classificação epidemiológica e o favorecimento da ocorrência de Leishmaniose Visceral em municípios que abrigam assentamentos de reforma agrária, pertencentes à Região de Saúde de Presidente Prudente-SP. As regiões de estudo foram classificadas segundo a estrutura do serviço de zoonoses e situação epidemiológica, aplicando-se análise espacial e tratamento cartográfico das informações. De 45 municípios, 19 (42,2\%) abrigam um total de 121 assentamentos, enfrentando diferentes cenários. Houve estreita relação quanto à disponibilização e atuação da equipe de zoonoses e a situação da Leishmaniose Visceral, especialmente nos municípios de estudo silenciosos (receptivos ou não) e em investigação. Os resultados contribuem para a vigilância em saúde, apoio a decisão em áreas prioritárias e fortalecimento do Sistema Único de Saúde (SUS).

Palavras-chave: assentamentos rurais, epidemiologia, risco, leishmaniose visceral, vigilância em saúde pública

\section{AGRARIAN REFORM SETTLEMENTS AND HEALTH SURVEILLANCE FOR VISCERAL LEISHMANIOSIS IN THE REGION OF PRESIDENTE PRUDENTE, SÃO PAULO, BRAZIL}

\begin{abstract}
The Visceral Leishmaniasis (VL) has interdependence with modified remnant forest regions, and the rural spaces tend to store vectors and reservoirs. This study aimed to establish relationships among risk categories according to epidemiological classification and favoring the occurrence of Visceral Leishmaniasis in counties that shelter agrarian reform settlements belonging to the to the Region of Health of Presidente Prudente-SP. The study regions were classified according to the category for zoonoses service and epidemiological situation, applying spatial analysis and cartographic treatment of the information. Of 45 counties, 19 (42.2\%) have a total of 121 settlements, facing different scenery. There was a close relationship between the availability and performance of the zoonoses team and Visceral Leishmaniasis situation, especially in the silent study counties (receptive or not) and in research. The results contribute to health surveillance, decision support in priority areas and strengthening of the Unified Health System (SUS). Keywords: rural settlements, epidemiology, risk, visceral leishmaniasis, public health surveillance.
\end{abstract}

\section{INTRODUÇÃO}

A Leishmaniose Visceral (LV) ou Calazar é uma enfermidade infecto-parasitária de notificação compulsória, ocasionada pelo agente etiológico Leishmania infantum (Leishmania (L.) chagasi) ${ }^{1}$. Pode acometer diferentes espécies de mamíferos, inclusive 0 homem ${ }^{2}$. Os cães domésticos (Canis familiaris) são considerados importantes reservatórios devido à convivência próxima dos seres humanos e elevada susceptibilidade à doença ${ }^{3}$. A transmissão ocorre principalmente por meio do repasto sanguíneo desempenhado por fêmeas de dípteros, pertencentes à família Psychodidae, 
popularmente conhecidos como flebotomíneos ${ }^{4}$. A espécie Lutzomyia longipalpis tem sido apontada como a principal responsável pela transmissão de $L$. infantum no Brasil ${ }^{5}$. Após inoculação no hospedeiro vertebrado, os parasitos apresentam tropismo por células do sistema mononuclear fagocitário (SFM), configurando infecções intracelulares obrigatórias ${ }^{6}$.

Na busca de uma construção diagnóstica plausível para a doença, é imprescindível que associe-se métodos parasitológicos, imunológicos ou moleculares com a sintomatologia e dados epidemiológicos ${ }^{3}$.

O diagnóstico pode ser efetuado através da confecção de lâminas de esfregaço para observação de formas amastigotas; cultura (in vivo e in vitro) para as formas promastigotas; exame histopatológico dos órgãos acometidos; utilização de Reação em Cadeia da Polimerase (Polymerase Chain Reaction - PCR) ou PCR quantitativo (Real Time PCR) para amplificação do ácido desoxirribonucleico (DNA) e isoenzimas para identificação de espécies do parasito ${ }^{7}$.

O Ministério da Saúde (MS) estabelece como método de triagem no diagnóstico da leishmaniose visceral canina (LVC) o teste rápido imunocromatografico (TR) DPP ${ }^{\circledR}$ BioManguinhos, realizado preferencialmente nos municípios ${ }^{7}$. A confirmação diagnóstica ocorre através do ensaio imunoenzimatico ELISA $^{\circledR}$ BioManguinhos em centros de controle de zoonoses (CCZs) municipais ou nos Laboratórios Centrais (LACENs) ${ }^{8}$.

No Estado de São Paulo (ESP), as ações desenvolvidas na esfera municipal para vigilância e controle da doença estruturam-se por meio de diretrizes do Programa de Vigilância e Controle da LV (PVCLV) ${ }^{9}$. É recomendado que os municípios realizem inquérito sorológico canino contemplando análises epidemiológicas fundamentadas na estratificação por risco ${ }^{10}$.

Segundo o PVCLV no ESP, a classificação epidemiológica deve basear-se em dois grupos distintos: municípios silenciosos (não possuem casos humanos e/ou caninos autóctones confirmados) e com transmissão (casos humanos e/ou caninos autóctones confirmados) ${ }^{9}$. Ainda, serão considerados nos municípios silenciosos a receptividade (presença ou não do vetor) e vulnerabilidade (chance de circulação de fontes de infecção) $)^{9,11}$.
De maneira geral, nas zonas urbanas, periurbanas e rurais, sabe-se que a dispersão da LV associa-se às mudanças das características ambientais, condições precárias de habitação da população e/ou ações antrópicas oriundas das ocupações territoriais ${ }^{9}$. Atrelado a estes fatores, somam-se parâmetros higiênico-sanitários e socioeconômicos $^{12}$. No entanto, essa zoonose possui forte interdependência com regiões de florestas remanescentes que foram modificadas, sendo os espaços rurais, importantes locais de armazenamento e concentração de vetores e reservatórios ${ }^{13}$. Nesse contexto, compreende-se a importância de estabelecer o processo de saúdedoença em regiões agrárias de organização familiar, como por exemplo, os assentamentos. Logo, essas unidades agrícolas merecem atenção especial em estudos de vigilância em saúde e assistência básica, pois configuram-se como possíveis focos de manutenção e facilitadores de rotas de dispersão de LV no território ${ }^{14}$.

O presente estudo objetivou dimensionar diferentes situações de risco epidemiológico conforme a classificação epidemiológica e o favorecimento da ocorrência de Leishmaniose Visceral em municípios que abrigam assentamentos de reforma agrária pertencentes à região de saúde de Presidente Prudente, São Paulo, Brasil.

\section{METODOLOGIA}

Realizou-se levantamento de dados dos assentamentos de reforma agrária instalados em municípios que integram a Rede Regional de Atenção à Saúde (RRAS) 11 - Presidente Prudente/SP através de fontes da Fundação Instituto de Terras do Estado de São Paulo (ITESP) "José Gomes da Silva" e do Instituto Nacional de Colonização e Reforma Agrária (INCRA). Esses municípios foram classificados pela sub-rede de leishmaniose do ESP, segundo a categoria para serviço de zoonoses compreendendo período até maio/2018: a) totalmente estruturado e funcionando (possui estrutura física própria, equipe capacitada e atuante para realização da triagem sorológica TR DPP ${ }^{\circledR}$ BioManguinhos); b) sem estrutura física e parcialmente funcionando (equipe capacitada e atuante, mas sem estrutura física própria e com realização da triagem sorológica TR DPP ${ }^{\circledR}$ BioManguinhos no Centro de Laboratórios Regional, Instituto Adolfo Lutz, Presidente Prudente V - CLR-IAL-PP V); c) sem estrutura física e sem funcionamento (possui 
equipe capacitada, mas não é atuante, sem estrutura física para realização da triagem sorológica TR DPP ${ }^{\circledR}$ BioManguinhos); d) sem serviço de zoonoses.

A classificação epidemiológica quanto a LV nos municípios de estudo foi realizada conforme o PVCLV no ESP em: municípios em investigação (MI); com transmissão canina (TC); transmissão humana e canina (THC); silenciosos receptivos e vulneráveis (SRV); silenciosos receptivos e não vulneráveis (SRNV); silenciosos não receptivos e não vulneráveis (SNRNV); silenciosos não receptivos e vulneráveis (SNRV) e com notificação de casos caninos, mas não receptivos (NCCNR), conforme a Figura 1.

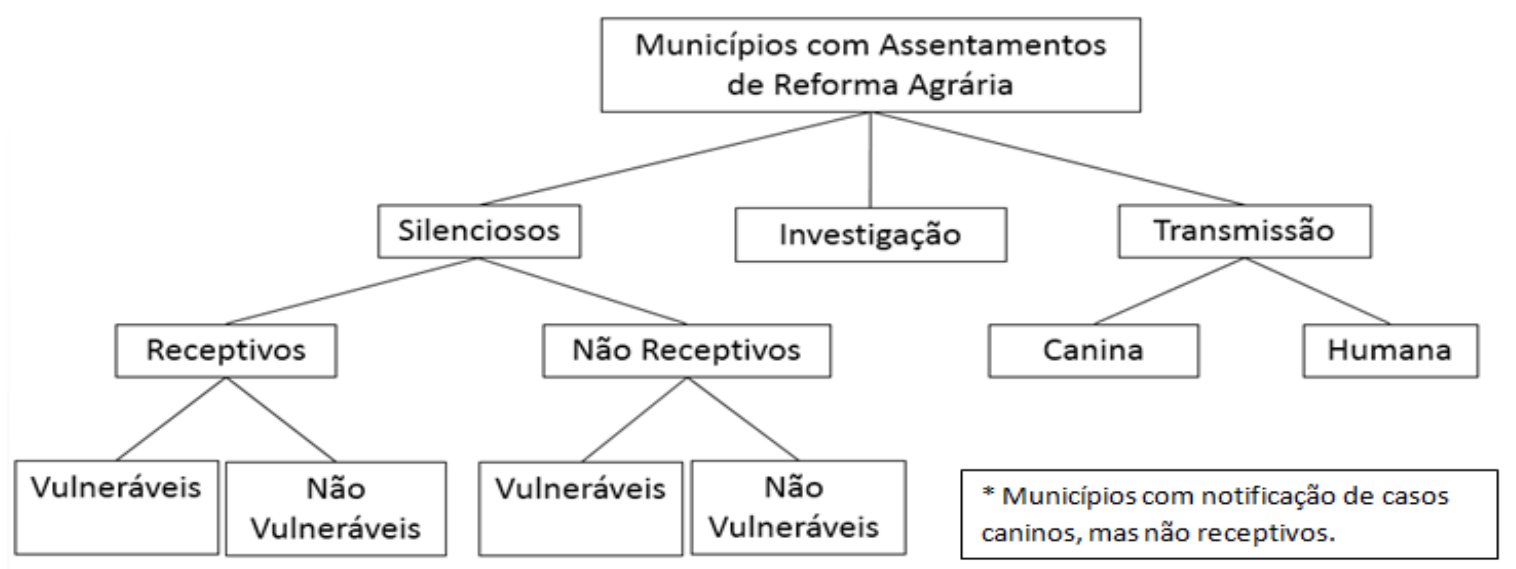

Figura 1. Classificação epidemiológica para os municípios da RRAS 11 Presidente Prudente/SP que possuem assentamentos de reforma agrária em seu território. Fonte: Adaptado do Manual do PVCLV no ESP (http://www.saude.sp.gov.br). Acesso em 16 de maio de 2018.

As informações sobre a disponibilidade de serviços de zoonoses e a classificação segundo a situação epidemiológica foram adquiridas através de fontes do Laboratório de Parasitologia do Núcleo de Ciências Biomédicas do CLR-IAL-PP $\checkmark$ que é referência regional para o diagnóstico da LV e da Superintendência do Controle de Endemias (SUCEN) - Presidente Prudente.

Para análise espacial e tratamento cartográfico da informação, os dados coletados nas etapas anteriores foram transpostos em tabelas de informação gráfica através de um Sistema de Informações Geográficas (SIG). Foram utilizadas bases de dados cartográficos disponibilizadas pelo Instituto Brasileiro de Geografia e Estatística (IBGE), pelo ITESP e INCRA. Os dados coletados foram convertidos em mapas temáticos, analisados por superfícies de interpolação através do software Arc Gis 10.3 do laboratório de Biogeografia da Universidade
Estadual Paulista (UNESP) "Júlio de Mesquita Filho", Campus de Presidente Prudente.

Os dados apresentados fazem parte de resultados parciais obtidos com o estudo avaliado e aprovado pelo Comitê Técnico Científico do Instituto Adolfo Lutz - CTC-IAL - 25H.2015, aprovado pelo Comitê de Ética em Pesquisa do IAL - CEPIAL, CAAE: 53247716.8.0000.0059, parecer №. 1.934.175 e pela Comissão de ética no Uso de Animais - CEUA- IAL -02/2016.

\section{RESULTADOS}

Dos 45 municípios existentes na área de abrangência da RRAS 11 - Presidente Prudente/SP, 19 (42,2\%) deles, abrigam um total de 121 assentamentos de reforma agrária. Sendo os municípios de Mirante do Paranapanema e Teodoro Sampaio detentores dos maiores números dessas unidades agrícolas em seus territórios, $35 \quad(28,9 \%)$ e $22 \quad(18,2 \%)$ respectivamente (Figura 2 ). 


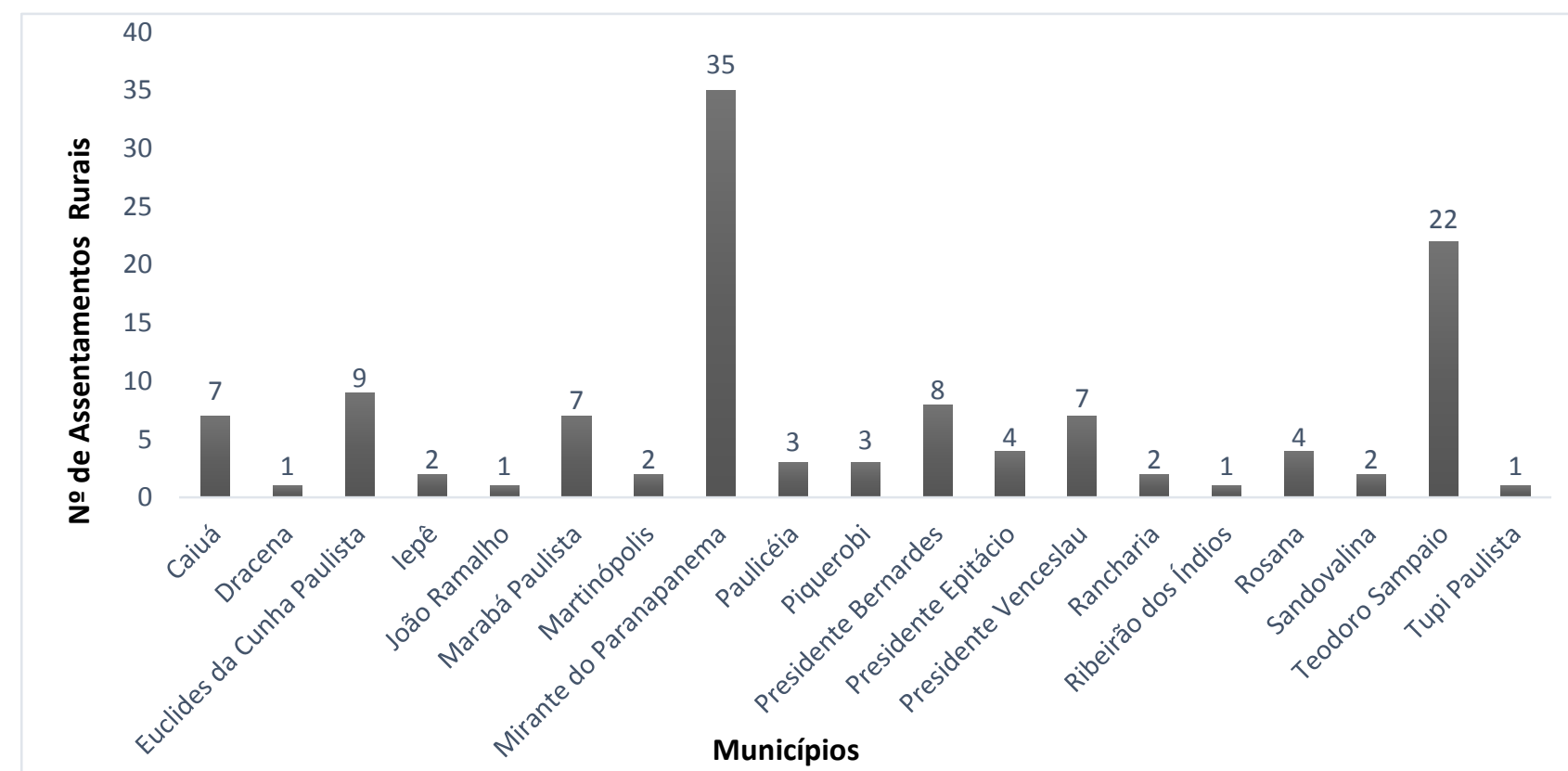

Figura 2. Distribuição dos municípios da RRAS 11 Presidente Prudente/SP com seus respectivos quantitativos de assentamentos de reforma agrária em seus territórios. Fonte: INCRA (http://www.incra.gov.br) e ITESP (http://www.itesp.sp.gov.br). Acesso em 16 de maio de 2018.

Dos 19 municípios com assentamentos, $16(84,2 \%)$ deles possuem pelo menos algum tipo de serviço de zoonoses, sendo $12(63,1 \%)$ totalmente estruturados, três $(15,8 \%)$ sem estrutura física, mas parcialmente funcionando e um $(5,3 \%)$ sem estrutura física e sem funcionamento, contando apenas com equipe capacitada, mas não atuante. Os municípios de Euclides da Cunha Paulista-SP, lepê-SP e RosanaSP não possuem nenhum tipo de serviço de zoonoses até o momento.

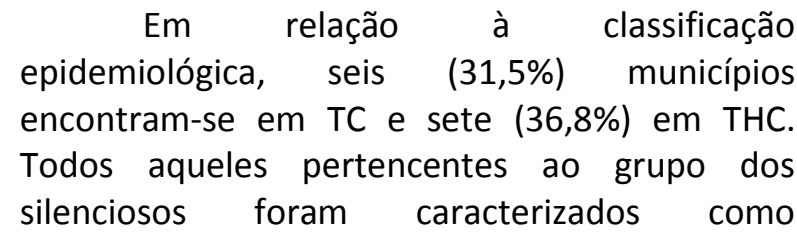
vulneráveis por proximidade a áreas endêmicas, sendo três $(15,8 \%)$ SNRV e um (5,3\%) SRV. Durante esse período, o município de Mirante do Paranapanema-SP estava sob investigação e João Ramalho-SP com NCCNR, conforme a Tabela 1 e Figura 3. 
Tabela 1. Distribuição dos municípios da RRAS 11 Presidente Prudente/SP que abrigam assentamentos de reforma agrária, classificados quanto à disponibilidade de serviços de zoonoses e a situação epidemiológica da Leishmaniose Visceral em seu território.

\begin{tabular}{lcc}
\hline Municípios & \multicolumn{1}{c}{$\begin{array}{c}\text { Serviço de } \\
\text { Zoonoses }\end{array}$} & $\begin{array}{c}\text { Situação } \\
\text { Epidemiológica }\end{array}$ \\
\hline 1. Caiuá & Totalmente Estruturado & TC \\
2. Dracena & Totalmente Estruturado & THC \\
3. Euclides da Cunha Paulista & Sem Serviço de Zoonoses \\
4. lepê & Sem Serviço de Zoonoses & Sem Estrutura Física, \\
& Parcialmente Funcionando & SNRV \\
5. João Ramalho & Totalmente Estruturado & NCCNR \\
6. Marabá Paulista & Totalmente Estruturado & THC \\
7. Martinópolis & Sem Estrutura Física, & TC \\
8. Mirante do Paranapanema & Parcialmente Funcionando & MI \\
& Totalmente Estruturado & THC \\
9. Paulicéia & Totalmente Estruturado & TC \\
10. Piquerobi & Sem Estrutura Física, & \\
11. Presidente Bernardes & Parcialmente Funcionando & TC \\
12. Presidente Epitácio & Totalmente Estruturado & THC \\
13. Presidente Venceslau & Totalmente Estruturado & THC \\
14. Rancharia & Totalmente Estruturado & TC \\
15. Ribeirão dos Índios & Totalmente Estruturado & TC \\
16. Rosana & Sem Serviço de Zoonoses & SNRV \\
17. Sandovalina & Sem Estrutura Física e & \\
18. Teodoro Sampaio & Sem Funcionamento & SRV \\
19. Tupi Paulista & Totalmente Estruturado & THC \\
\hline
\end{tabular}

Fontes: Laboratório de Parasitologia do Núcleo de Ciências Biomédicas do CLR - IAL - PP V e SUCEN - Presidente Prudente, 2018. Legenda: MI (Município em Investigação); TC (Transmissão Canina); THC (Transmissão Humana e Canina); NCCNR (Notificação de Casos Caninos, mas Não Receptivo); SRV (Silencioso Receptivo Vulnerável); SNRV (Silencioso Não Receptivo Vulnerável).

\section{DISCUSSÃO}

A RRAS 11 - Presidente Prudente/SP possui grande quantidade de assentamentos de reforma agrária, contando atualmente com 121 deles, distribuídos em 19 municípios. Ao todo, compreendem mais de 6.046 lotes em uma área de $144.896,71 \mathrm{ha}^{15,16}$. Em estudos prévios, foram comprovados casos autóctones de transmissão de LVC no assentamento Santa Rita, no município de Tupi Paulista-SP e durante a caracterização ambiental dos lotes do assentamento Santa Terezinha da Água Sumida no município de Teodoro Sampaio-SP, foram evidenciados cenários propícios para manutenção do ciclo da doença, como a existência de currais, galinheiros e chiqueiros próximos às residências; acúmulo de lixo e matéria orgânica; áreas de vegetação Colloq Vitae 2018 set-dez; 10(3): 13-20. DOI: 10.5747/cv.2018.v10.n3.v238 ISSN 1984-6436/@ 2018 - Publicado pela Universidade do Oeste Paulista. Artigo Open Access sob uma licença CC BY-NC-ND (http://creativecommons.org/licenses/by-nc-nd/4.0/) margeando vários lotes e extensas áreas de pastagens combinadas a cães soltos circulando livremente, criando um ambiente favorável para reservatórios silvestres ${ }^{12,17,18}$. Ainda, soma-se a expansão do plantio de monoculturas, como a cana-de-açúcar, outro fator contribuinte para o efeito de radiação da zoonose em vários desses espaços ${ }^{14}$, pois muitos cães com LVC oriundos de áreas urbanas são abandonados nesses canaviais próximos aos assentamentos, porque os proprietários não querem que seus animais sejam eutanasiados, conforme recomendação do PVCLV ${ }^{17}$.

É importante salientar que os assentamentos de reforma agrária não obedecem necessariamente a um padrão de organização e não devem ser tratados como regiões 
marginalizadas. Porém, do ponto de vista sanitário, possuem grande potencial para albergar reservatórios de LV, merecendo atenção especial dos gestores municipais por configurarem regiões prioritárias de risco, necessitando ações de vigilância em saúde e de disponibilização e atuação de equipe de zoonoses capacitada ${ }^{12}$. Nesse contexto, três municípios de estudo $(15,8 \%)$ se destacam: dois localizados na região do Pontal do Paranapanema (Rosana-SP e Euclides da Cunha Paulista-SP) e um no Alto do Capivari (lêpe-SP), todos sem serviços de zoonoses e classificados como áreas silenciosas não receptivas para $o$ vetor flebotomíneo. Conforme recomendações do PVCLV, mesmo os estados e municípios sem notificações de casos humanos e/ou caninos de LV devem adotar ações de vigilância, evitando ou reduzindo os impasses relacionados a essa zoonose, principalmente em regiões vulneráveis. Esse fato também deve aplicar-se ao município de Sandovalina-SP, classificado como SRV e que apesar de possuir equipe capacitada, esta não é atuante.
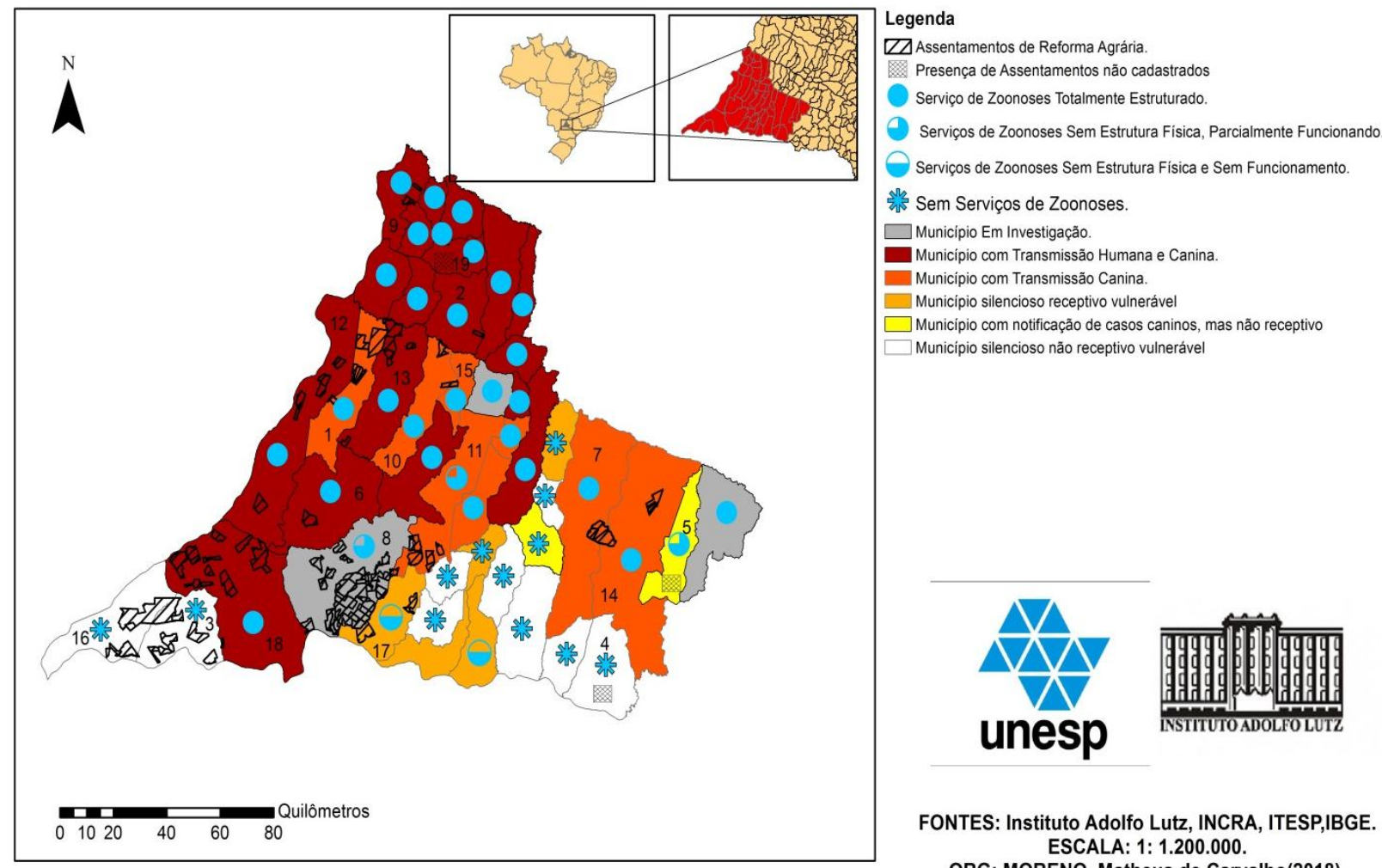

FONTES: Instituto Adolfo Lutz, INCRA, ITESP,IBGE. ESCALA: 1: 1.200 .000

ORG: MORENO, Matheus de Carvalho(2018) DATUM: SIRGAS 2000.

Figura 3. Situação Epidemiológica da Leishmaniose Visceral, disponibilidade de Serviços de Zoonoses e localização dos Assentamentos de Reforma Agrária nos municípios que compõem a Região de Saúde de Presidente Prudente - São Paulo - Brasil, em Maio/2018

O município de João Ramalho-SP é o único que apresentou NCCNR, possuindo equipe capacitada e atuante, mas sem estrutura física própria e com realização da triagem sorológica TR DPP ${ }^{\circledR}$ BioManguinhos no CLR - IAL - PP V. São necessárias a continuidade do trabalho de campo e compreensão da existência de ações nesse local para a captura e o estudo da fauna de flebotomíneos, elucidando se de fato é uma área não receptiva, já que existem casos autóctones notificados de LVC.
Os $68,4 \%$ dos municípios de estudo que encontram-se em transmissão humana e/ou canina (Caiuá-SP, Dracena-SP, Marabá PaulistaSP, Martinópolis-SP, Paulicéia-SP, Piquerobi-SP, Presidente Bernardes-SP, Presidente Epitácio-SP, Presidente Venceslau-SP, Rancharia-SP, Ribeirão dos Índios-SP, Teodoro Sampaio-SP e Tupi Paulista-SP), possuem algum tipo de serviço de zoonoses, carecendo da realização ou continuação de medidas pertinentes após essa estratificação epidemiológica, incluindo a avaliação das condições sanitárias, manejo 
ambiental, levantamento entomológico, limpeza dos ambientes, medidas de educação em saúde, entre outros ${ }^{9}$. As ações devem ser efetivadas de maneira integrada e de forma hábil o suficiente para prevenir ou conter o agravo em sua completitude ${ }^{9,10}$.

Outro ponto que merece atenção especial é em relação aos municípios silenciosos, receptivos ou não (Euclides da Cunha Paulista-SP, Rosana-SP e Sandovalina-SP) e em investigação (Mirante do Paranapanema-SP). Essas seriam de fato áreas que se configuram com risco eminente, onde não há notificações de casos autóctones humanos e/ou caninos ou não possuem um serviço de vigilância estruturado para notificações do referido agravo, visto que não contam com serviço de zoonose com estrutura física funcionando. Diante do exposto, os próximos passos seriam levar em consideração a existência da realização de inquéritos sorológicos focais nesses locais (especialmente nas zonas rurais e assentamentos de reforma agrária), se os proprietários de animais procuram serviço especializado para o diagnóstico canino e, por conseguinte, se esse é notificado ao serviço público ${ }^{7}$.

A diversidade da ocupação territorial no Oeste Paulista torna a compreensão da LV complexa e repleta de particularidades. Trabalhar apenas com as variáveis diagnóstico e tratamento de doentes não é o suficiente para o entendimento do processo saúde-doença. Nesse aspecto, a aplicação da espacialidade através de métodos cartográficos é fundamental. Outros estudos poderão ser direcionados às escalas

\section{REFERÊNCIAS}

1. Centers for Disease Control and Prevention. Parasites-Leishmaniasis, 2013. Disponível em: <http://www.cdc.gov/parasites/Leishmaniasis/ biology.html >. Acesso em: 24 abr 2018.

2. Batista FMA, Machado FFOA, Silva JMO, Mittmann J, Roxo BP, Ribeiro SA. Leishmaniose: perfil epidemiológico dos casos notificados no estado do Piauí entre 2007 e 2011. Rev Univap. 2014;20(35):44-55.

DOI:

http://dx.doi.org/10.18066/

revunivap.v20i35.180

3. Borja LS. Estudo da correlação entre a carga parasitária de cães com diferentes apresentações clínicas da Leishmaniose Visceral e a transmissão locais em áreas urbanas e seus respectivos assentamentos de reforma agrária, visando à realização de ações integradas e efetivas para a prevenção e controle da doença nesses espaços.

\section{CONCLUSÕES}

Conclui-se que há uma estreita relação quanto ao tipo de disponibilização de serviço de zoonoses, atuação da equipe zoonoses e a situação epidemiológica para a LV, especialmente nos municípios de estudo silenciosos (receptivos ou não) e em investigação. Com os resultados obtidos também é possível dimensionar diferentes situações de risco epidemiológico para a doença, auxiliar no trabalho das entidades envolvidas na vigilância em saúde e apoiar na tomada de decisão em áreas prioritárias, principalmente a importância da realização de ações de vigilância e controle em assentamentos de reforma agrária.

É necessário que lacunas sobre dados epidemiológicos e outros aspectos relevantes sejam compreendidos e entendidos na totalidade dos territórios, tanto em áreas urbanas, periurbanas e rurais dos municípios. Contribuindo para o fortalecimento do Sistema Único de Saúde (SUS) e na equidade de serviços prestados para as populações vigentes.

\section{CONFLITOS DE INTERESSE}

Os autores declaram não haver qualquer potencial conflito de interesses que possa interferir na imparcialidade deste trabalho científico.

ao vetor da Leishmania infantum. [Tese]. Salvador: Universidade Federal da Bahia - Centro de Pesquisas Gonçalo Moniz/FIOCRUZ, 2013.

4. Gontijo CMF, Melo MN. Leishmaniose visceral no Brasil: quadro atual, desafios e perspectivas. Rev Brasil Epidemio. 2004;7(3):338-349. DOI: http://dx.doi.org/10.1590/S1415790X2004000300011

5. Silveira FT, Lima LVR, Santos TV, Ramos PKS, Campos MB. Revendo a trajetória da leishmaniose visceral americana na Amazônia, Brasil: de Evandro Chagas aos dias atuais. Rev Pan-Amaz Saude. 2016;7(Esp):15-22. DOI: http://dx.doi.org/10.5123/s217662232016000500003 
6. Gomes KWP, Benevides NA, Vieira FJF, Burlamaqui MPM, Vieira MAP, Fontenelle LMAR. Leishmaniose tegumentar em paciente com espondilite anquilosante utilizando adalimumabe. Rev Bras Reumatol. 2012;52(3):447-452. DOI: http://dx.doi.org/10.1590/S0482$\underline{50042012000300014}$

7. D'Andrea LAZ et al. Leishmaniose Visceral: implantação do teste rápido $\mathrm{DPP}^{\circledR}$ BioManguinhos nos inquéritos sorológicos canino na área de abrangência do Instituto Adolfo Lutz de Presidente Prudente-SP. In: Dias LS, Guimarães RB. (Org.). Desafios da saúde ambiental. Tupã: ANAP, 2015. p. 126-140. Disponível em: <http://www.amigosdanatureza.org.br/biblioteca /livros/categoria/ cod/45>. Acesso em: 21 jun 2018.

8. Brasil. Ministério da Saúde. Secretaria de Vigilância em Saúde. Departamento de Vigilância das Doenças transmissíveis. Nota Técnica conjunta no 1/2011 CGDT-CGLAB/DEVIT/SVS/MS, 2011.

9. São Paulo. Manual de vigilância e controle da leishmaniose visceral americana do Estado de São Paulo. Secretaria de Estado da Saúde, Superintendência de Controle de Endemias SUCEN e Coordenadoria de Controle de Doenças - CCD, 2006. Disponível em: http://www.saude.sp.gov.br/resources/sucen/ho mepage/downloads/arquivos-leishmaniosevisceral-americana/manual Iva.pdf. Acesso em: 16 jul 2018.

10. Von Zuben APB, Donalísio MR. Dificuldades na execução das diretrizes do Programa de Vigilância e Controle da Leishmaniose Visceral em grandes municípios brasileiros. Cad Saúde Púb. 2016;32(6): $\quad$ e00087415. DOI: http://dx.doi.org/10.1590/0102-311X00087415

11. Rangel $O$ et al. Classificação epidemiológica dos municípios segundo o Programa de Vigilância e Controle da Leishmaniose Visceral Americana no estado de São Paulo, para 2013. BEPA. 2013;10(111):3-14.

12. D'Andrea LAZ. Leishmaniose visceral na região de Presidente Prudente, São Paulo: distribuição espacial e rotas de dispersão. [Tese]: Doutorado em Geografia - Universidade Estadual Paulista, Presidente Prudente, 2017.

13. Silva FS. Patologia e patogênese da leishmaniose visceral canina. Rev Trop Ciên Agr Biol. 2007;1(1):20-31. DOI: https://www.researchgate.net/publication/2200 $\underline{00420}$

14. D'Andrea LAZ, Guimarães RB. A importância da análise de distribuição espacial da leishmaniose visceral humana e canina para as ações de vigilância em saúde. Hygeia. 2018;14(28):121-138. DOI: http://dx.doi.org/10.14393/Hygeia142810

15. Instituto Nacional de Colonização e Reforma Agrária (INCRA). Disponível em: http://www.incra.gov.br/. Acesso em: 26 abr 2018.

16. Fundação Instituto Terras do Estado de São Paulo (ITESP). Disponível em: http://www.itesp.sp.gov.br. Acesso em: $28 \mathrm{abr}$ 2018.

17. D'Andrea LAZ, Vicentini CF, Samizava EY, Guimaraes RB. Leishmaniose visceral no meio rural de Teodoro Sampaio/SP/Brasil: uma nova fronteira. In: VII Simpósio Nacional de Geografia da Saúde e IV Fórum Internacional de Geografia da Saúde. Brasília: UnB. 2015;(7):120-130.

18. D'Andrea LAZ, Gesse LE, Flores EF, Tsuda SK, Guimaraes RB. Leishmaniose visceral na área urbana e rural de Tupi Paulista - SP/Brasil entre 2006 e 2015. In: VIII Simpósio Nacional de Geografia da Saúde - V Fórum Internacional de Geografia da Saúde, 2017, Dourados - Mato Grosso do Sul. Anais VIII GeoSaúde. Saúde na Fronteira e Fronteira(s) na Saúde. Dourados: UFGD. 2017; 342-355.

Recebido para publicação em 17/08/2018

Revisado em 27/08/2018

Aceito em 03/09/2018 